\title{
ADAPTIVE OPTICS: PERFORMANCE AND LIMITATIONS
}

\author{
FRANCOIS RODDIER \\ Institute for Astronomy, University of Hawaii \\ 2680 Woodlawn Drive, Honolulu, HI 96822
}

January 7, 1993

\begin{abstract}
Adaptive optics systems are now being developed for astronomical applications. On large telescopes, a substantial image improvement can be obtained in the near infrared, using natural guide stars.
\end{abstract}

Key words: Adaptive optics - Astronomy - High angular resolution - Seeing

\section{Introduction}

Adaptive optics (AO) is a means for real-time compensation of atmospherically induced wavefront errors. A reference source or "guide star" must be available to sense the wavefront errors. It can be either natural or artificial. Most currently planned astronomical AO systems use natural reference sources and divert part of the light from the sky to a wavefront sensor. There are essentially three beam splitting techniques: intensity splitting using a beam splitter, spectral splitting using a dichroic mirror to sense the wavefront outside of the bandpass of scientific interest, and spatial splitting using a hole in a mirror to extract the sensor light from a nearby star. A control system is used to convert data from the wavefront sensor into voltages which are applied to an actively controllable deformable mirror.

First proposed by Babcock (1953) for astronomical applications, AO has been widely developed for military applications. Progress in computers and detectors makes the technique now adaptable to astronomy. Rather than describing the various technical options, this paper discusses the fundamental limitations of the technique, presents astronomical results obtained so far and the performance one can expect in a near future. Because artificial guide star technology is still in its infancy, the discussion is mainly limited to AO systems based on the use of natural guide stars.

\section{Image quality criteria}

Before discussing performance, one must first define performance criteria. Astronomers are used to define image quality in terms of image full width at half maximum (fwhm). Images produced by AO systems require the use of other criteria. Fig. 1 shows an example of intensity distribution recorded in a stellar image with and without wavefront compensation. These 30-second exposure CCD images of Arcturus were taken at $0.85 \mu \mathrm{m}$ with the 3.6-m CFH telescope and the prototype AO system built at the University of Hawaii (UH). The uncompensated image has a 0.4 arc-second fwhm. It was taken under good Mauna Kea seeing conditions with static voltages applied to the deformable mirror to compensate for both telescope and system aberrations. The compensated image shows a sharp peak of intensity but large wings. Its fwhm is 0.08 arc-second, which makes it the sharpest long exposure stellar image ever taken by a ground-based telescope. Although this is close 

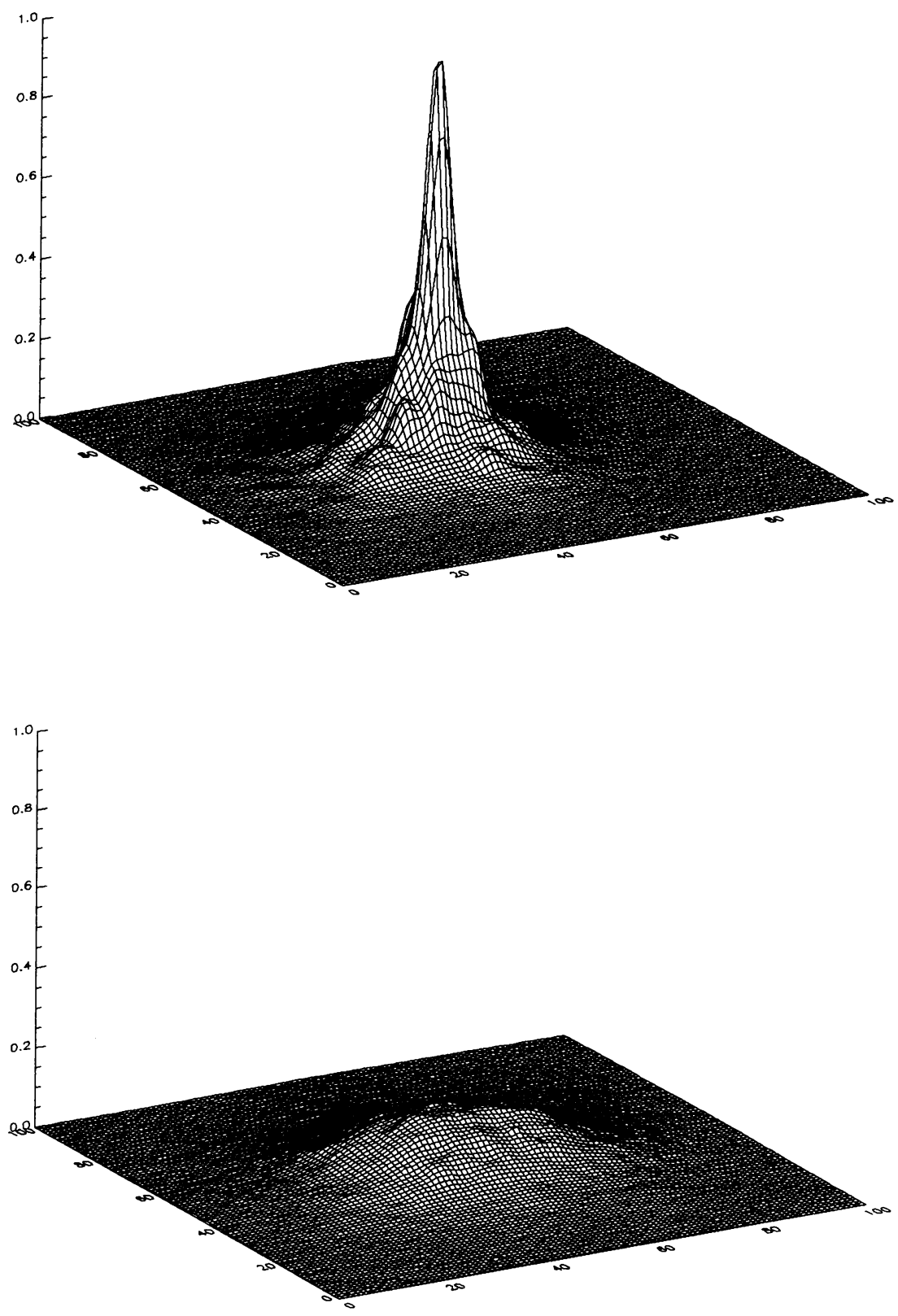

Fig. 1. Long exposure CCD images of Arcturus recorded at $0.85 \mu \mathrm{m}$ with the 3.6-m CFH telescope. Compensated (top) and uncompensated (bottom) image. The field of view is $1.4 \times 1.4$ arc-second. 
to the fwhm of the diffraction-limited image, this image is still far for being diffraction limited. A criterion widely used for AO systems is the Srehl ratio, or ratio of the maximum intensity in the point source image to that in the diffraction-limited image. The Strehl ratio of the compensated image shown in Fig. 1 is only 0.1. Other possible criteria are the diameter for $50 \%$ encircled energy, or the fraction of light in the diffraction-limited core. In the following we shall use the normalized Strehl ratio, that is the Strehl ratio normalized to unity for an uncompensated image recorded with an infinitely large telescope.

\section{Performance limitations}

The main limitations of adaptive optics systems are:

1. the wavefront fitting error due to the finite number of degrees of freedom of the AO system,

2. the time delay error due to the finite loop bandwidth,

3. the sensor noise or, assuming an ideal detector, the photon shot noise,

4. the isoplanicity error, when the direction of the object and of the guide star do not coincide.

In addition, one may have chromatic errors when observations and sensing are done at different wavelengths, and amplitude errors due to scintillation effects.

The brightness and angular distance of the available guide star essentially dictates the number of independent parameters one can safely estimate on the wavefront, hence the amount of image improvement one can expect to achieve. Fig. 2 shows how the residual mean square wavefront errors decreases as a function of the number of free parameters (or modes) used in the compensation. In all cases, the first two modes are assumed to be wavefront tip and tilt. Higher order compensation can be either zonal (dotted curve) or modal. The dashed line is for Zernike modes, the full line for the atmospheric Karhunen-Loeve modes which theoretically represent the most efficient compensation. In the following we describe the amount of compensation as an equivalent number of compensated Zernike terms.

\section{Guide star requirements}

Fig. 3 shows the guide star limiting magnitude and maximum distance to object for different levels of compensation indicated by the equivalent number of compensated Zernike terms. It also shows contour lines based on the Bahcall and Soneira Galaxy model, indicating the probability of finding the appropriate guide star. Full lines are for a 30 degree Galactic latitude and a 90 degree longitude. The dotted line is the $50 \%$ probability contour for the Galactic pole. AO systems developed for military applications are not considered here, because there limiting magnitude is too low for these systems to be useful in astronomy. Although based on military technology, the French "COME-ON" system (Rigaut et al., 1991) was built for astronomy and uses an electron-bombarded CCD (EBCCD) which enhances its sensitivity. The COME-ON+ system (Gendron et al., 1991) is an upgraded version currently being tested. Fig. 3 shows that on on a 3.6-m telescope, the probability of finding an appropriate guide star is still low (less than $10 \%$ ), unless compensation is limited to 


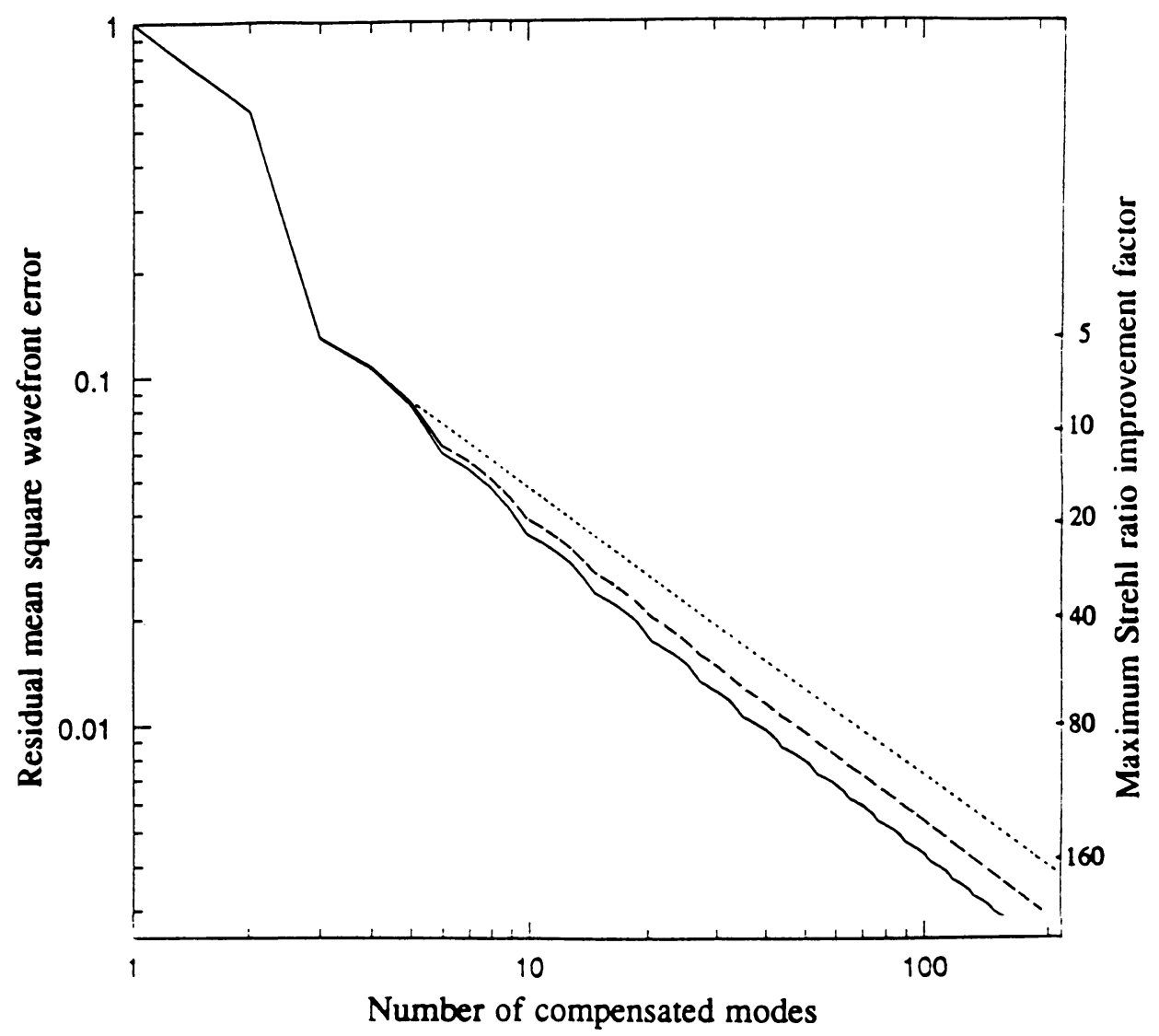

Fig. 2. Residual mean square wavefront error as a function of the number of compensated modes. Dotted line: zonal compensation. Dashed line: Zernike modes. Full line: Karhunen-Loeve modes.

wavefront tip/tilt compensation (first two Zernike terms). The UH system (Roddier et al., 1991) is based on the innovative concept of wavefront curvature sensing and compensation (Roddier, 1988) and owe its high performance to the use of an array of discrete detectors, photon counting avalanche photodiodes (APDs). On an 8-m telescope, the UH system should be able to provide a compensation equivalent to 9 Zernike terms (up to 3 rd degree) over $80 \%$ of the sky ( $50 \%$ at the Galactic pole).

\section{Strehl ratio improvement}

It is clear from Fig. 3 that, even on large telescopes, the use of natural guide stars will most of the time limit the amount of compensation to a few tens of Zernike terms. Fig. 4 shows how, for a given amount of compensation, the normalized Strehl ratio (defined in section 2) varies as a function of the ratio $D / r_{0}$ of the 


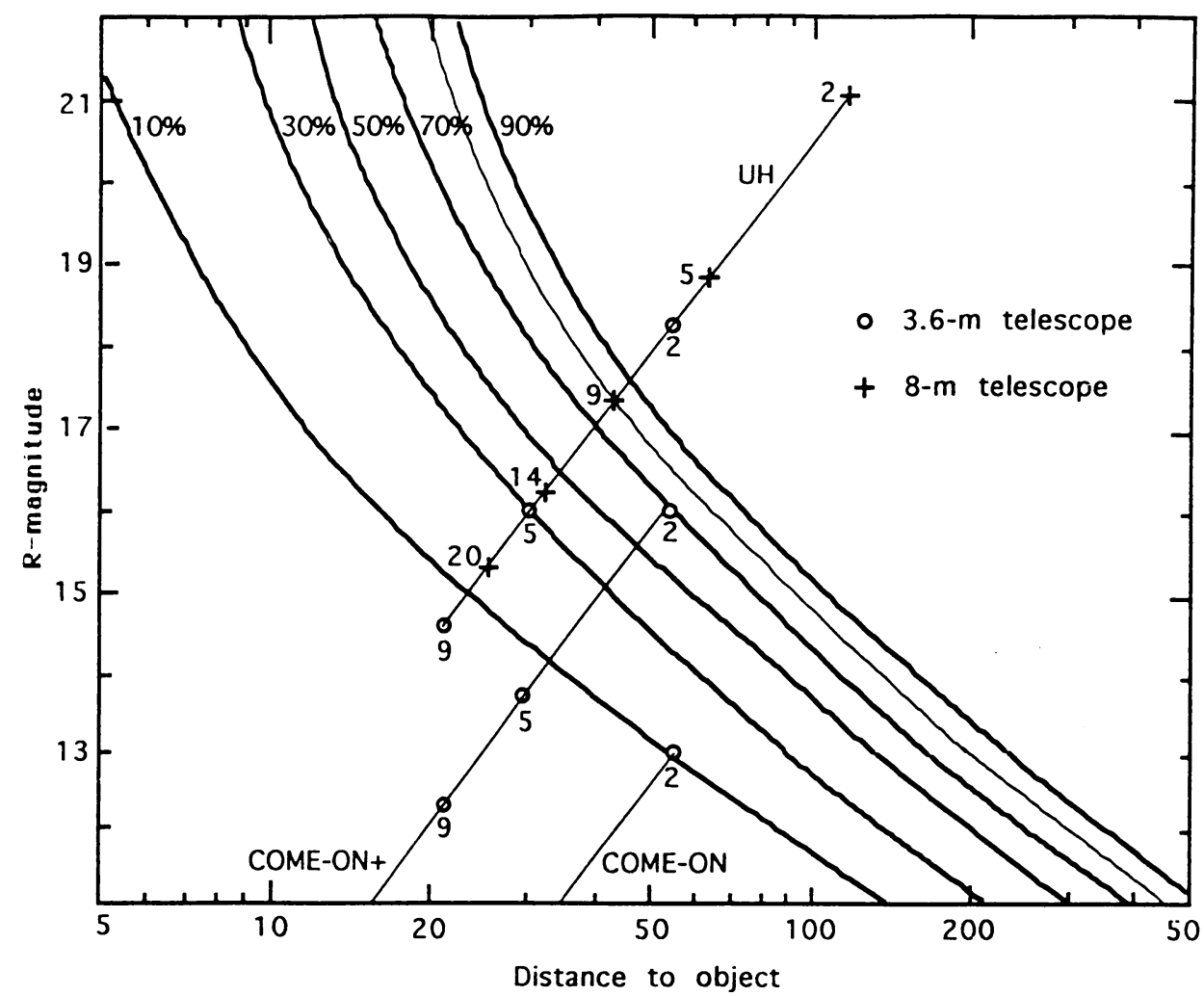

Fig. 3. Guide star requirements for different numbers of compensated Zernike terms and different AO systems. The contour lines indicate the probability of finding a satisfactory guide star.

telescope diameter $D$ over Fried's seeing parameter $r_{0}$. It shows that for, a given amount of compensation, there is an optimum aperture size or equivalently and optimum wavelength at which the highest resolution is achieved. For a compensation equivalent to 9 Zernike terms, the maximum resolution is achieved for $D / r_{0}=8$. Under average seeing conditions, this occur in the $J$ band for a 4-m class telescope and in the $\mathrm{K}$ band for an 8-m telescope. At this optimum wavelength, the Strehl ratio achieved is of the order of 0.3 . Higher Strehl ratios are achieved at longer wavelengths.

Clearly diffraction-limited imaging in the visible with a large telescope cannot rely on natural guide stars. Two different approaches can be envisaged, artificial guide stars or a combination of adaptive optics with interferometry. These options are discussed in the next two sections. 


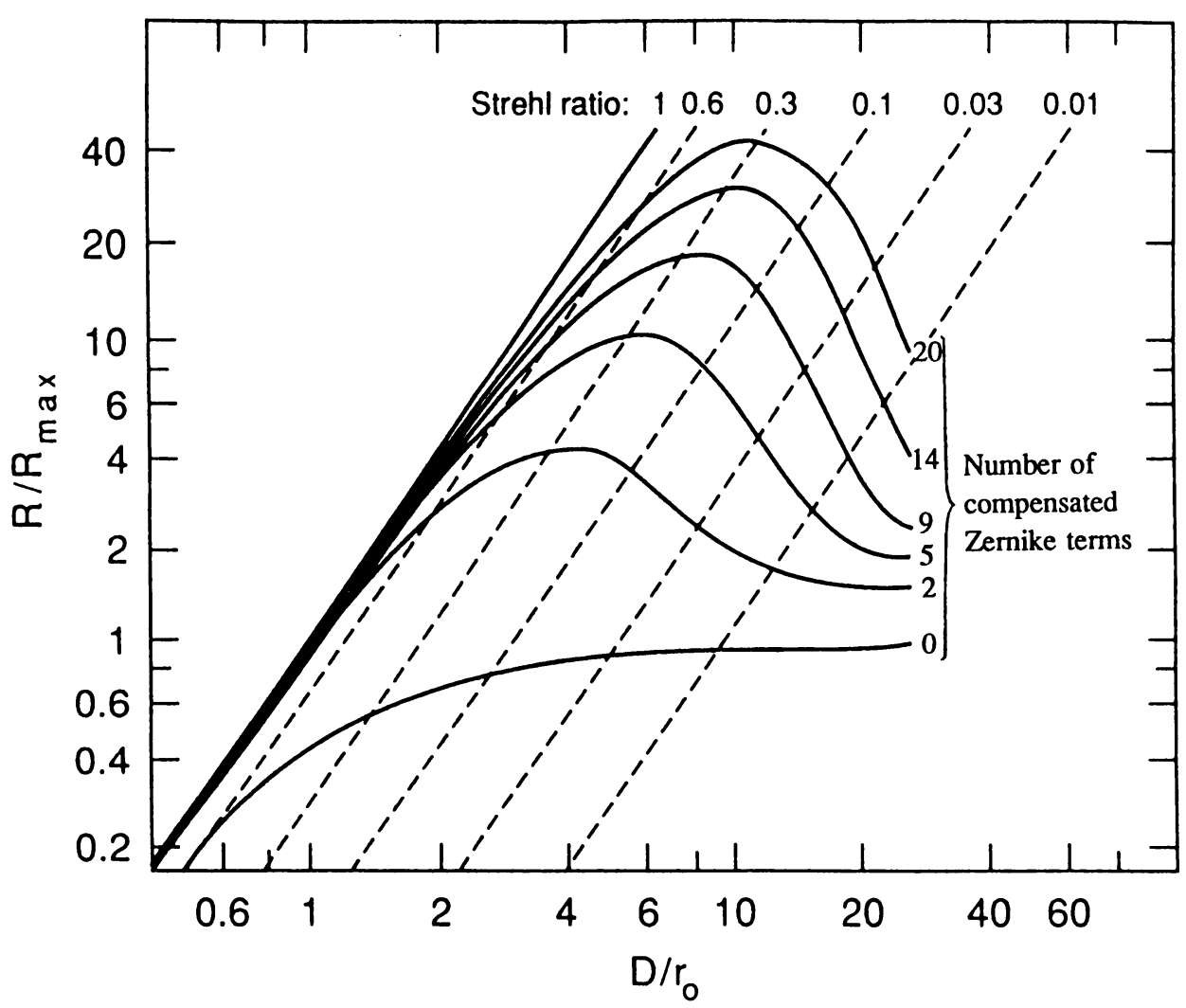

Fig. 4. Normalized Strehl ratio as a function of $D / r_{0}$ for different number of compensated Zernike modes.

\section{Artificial guide stars}

Because of the lack of natural reference sources, artificial guide stars have been produced by atmospheric backscattering of laser light. The technique is now declassified, but its application to astronomy presents some difficulties. Because the artificial beam goes twice through the atmosphere, the wavefront tilts cancel out. One still has to rely on natural stars to control image motion. Achieving diffractionlimited imaging in the visible, still requires a bright reference star unless a second laser beam and AO system is used to sharpen also the natural guide star (Rigaut and Gendron, 1992). Because the artificial light source is produced inside the atmosphere, rather than at infinity, a cause of errors known as focus anisoplanatism appear in the compensation (Fried, 1992). Even when using high altitude backscatter from the natural atmospheric sodium layer, several laser beams appear necessary to compensate an 8-m telescope. Finally high power lasers are expensive to purchase and to maintain, they require aircraft safety measures, and produce light pollution 
against which astronomers have long been fighting. Because of these difficulties, we believe that most astronomical AO systems will rely on natural guide stars, at least for the next coming years.

\section{Combination with interferometry}

Partial adaptive optics compensation has also been considered as a means to enhance high angular resolution interferometry. Conan et al. (1992) discussed the SNR performance of speckle imaging with partial adaptive optics correction, assuming pure photon noise but neglecting its effect on the wavefront sensor. Partial wavefront compensation reduces the number of speckles in the image and therefore increases the signal-to-noise ratio in the image reconstruction. There is a critical $D / r_{0}$ value below which simple deconvolution of a long exposure becomes the best approach. Partial wavefront compensation is also expected to enhance the performance of a long baseline interferometer (Rousset et al., 1991; Loos, 1992). In this case, one must also control the wavefront "piston" mode, that is combine AO with active fringe tracking. For small values of $D / r_{0}$ on can expect to record long exposure fringes with a visibility approximately given by the Strehl ratio in a compensated long exposure image. For larger $D / r_{0}$ values, one still has to rely on short exposures, albeit with an increased sensitivity.

\section{Astronomical results}

Although currently limited to bright objects, AO has already produced significant astronomical results. These results were essentially obtained in Europe with the COME-ON AO system (Rigaut et al., 1992). They were obtained from images taken in the J, H, K, L' and M bands. Among observed objects are,

1. $\eta$ Carinae: the high resolution images reveal a lumpy asymmetric dust distribution in a plane perpendicular to the mass ejection axis.

2. Ceres: a temperature map was obtained revealing its rotation axis.

3. $\zeta$ Canis Majoris: images show a condensation around this young stellar object. The condensation is clearly elongated in a direction perpendicular to the optical jet.

AO appears to be especially well suited to the study of young stellar objects. The COME-ON+ and UH systems are soon expected to considerably extend the number of objects accessible to $\mathrm{AO}$.

\section{Conclusion}

Application of AO to astronomy is making rapid progress and many observatories are now considering ways to equip their telescopes with an AO system. The technique is still considered as expensive, however progress have been made towards lower cost systems (Roddier, 1992), and it is our belief that not only telescopes, but even independent astronomical instruments (cameras and spectrographs) will soon be equipped with built-in AO systems able to work with faint natural guide stars. The technique will also undoubtedly enhance the performance of the long baseline optical interferometers currently under construction. 


\section{References}

Babcock H.W.: 1953, "The possibility of compensating astronomical seeing", Pub. Astr. Soc. Pac. 65, 221-224.

Conan J.M., Madec P.Y., Rousset G.: 1992, "Evaluation of image quality obtained with adaptive optics partial correction", Proceedings ESO Conference on Progress in Telescope and Instrumentation Technologies, edit. Ulrich, M.H.: ESO, Garching bei Munchen, in press.

Fried D.L.: 1992, "Analysis of focus anisoplanatism: the fundamental limit in performance of artificial guide star adaptive optics systems", Proceedings of Laser Gride Star Adaptive Optics Workshop, edit. Fugate R.Q.: Phillips Lab., Kirtland Air Force Base, N.M., 37-80.

Gendron E., Cuby J.C., Rigaut F., Lena P., Fontanella J.C., Rousset G., Gafiard J.P., Boyer C., Richard J.C., Vittot M., Merkle F., Hubin N.: 1991, "The COME-ON-PLUS project: An upgrade of the COME-ON adaptive optics prototype system", Proceedings SPIE Conference 1542, 297-307.

Loos G.C.: 1992, "On the implementation of adaptive optics for improving broadband spectral response in Michelson Interferometry", in OSA Topical Meeting on Adaptive Optics for Large Telescopes, Technical Digest Series9, 76-78.

Rigaut F., Rousset G., Kern P., Fontanella J.C., Gaffard J.P., Merkle F., and Lena P.: 1991, "Adaptive Optics on a 3.6-m telescope: results and performance", Astron. Astrophys. 250, 280-290.

Rigaut F. and Gendron E.: 1992, "Laser guide star in adaptive optics: the tilt determination problem", Astron. Astrophys. 261, 677-684.

Rigaut F., Combes M., Dougados C., Lena P., Mariotti J.M., Saint-Pe O., Alloin D., Mallet F., Bertout C., Gallais P., Gehring G.: 1992, "Astrophysical results with COME-ON", Proceedings ESO Conference on Progress in Telescope and Instrumentation Technologies, edit. Ulrich, M.H.: ESO, Garching bei Munchen, in press.

Roddier F.: 1988, "Curvature sensing and compensation: a new concept in adaptive optics", Appl. Opt. 27, 1223-1225.

Roddier F., Northcott M., Graves J.E.: 1991, "A simple low-order adaptive optics system for near-infrared applications", Pab. Astron. Soc. Pac. 103, 131-149.

Roddier F.: 1992, "Towards lower cost adaptive optics systems", in OSA Topical Meeting on Adaptive Optics for Large Telescopes, Technical Digest Seriese, 59-62.

Rousset G., Madec P.Y., Rabaud D.: 1991, "Adaptive optics partial correction simulation for two telescope interferometry", Proceedings ESO Conference on High Resolution Imaging by Interferometry II, edit. Beckers J.M. and Merkle F.: ESO, Garching bei Munchen, in press.

\section{Discussion:}

Eckart:

Could you say something concerning the calibration of the AO images, i.e. how well can you calibrate the underlying seeing background in the case of stars with faint extended emission halos? What will the speckle seeing statistics be in case of partial correction and the combination of $\mathrm{AO}$ with speckle techniques?

Roddier:

Because of seeing variations, the calibration of AO images is expected to be difficult, as it is now for speckle interferometry. For some applications one may be able to model anisoplanicity effects and rely on nearby stars within the field of view. For other applications, one may be able to use non-redundant imaging techniques to calibrate long exposures. 


\section{Zinnecker:}

Can you say something about the wavelength dependence of the isoplanatic angle? Roddier:

The isoplanatic patch size depends essentially on the degree of compensation achieved (equivalent number of Zernike or Karhünen-Loéve modes). In this sense it is independent of wavelength and - to a first approximation - of seeing conditions. Typically, on a 4-m telescope the radius varies from 1 arcminute for tip/tilt compensation down to 20 arcsecond for about 10 modes and only a few arcsecond for about 100 modes. Because the degree of compensation necessary to achieve a given Strehl ratio increases with decreasing wavelength, the isoplanatic patch size decreases accordingly.

\section{Haniff:}

When discussing the combination of $\mathrm{AO}$ and post-detection processing you mentigned a critical value of $\mathrm{D} / \mathrm{r}_{0}$ beyond which post-detection processing would be yseful. What is this critical value?

\section{Roddier:}

I am referring to calculations made by Gérard Rousset and his colleagues at ONERA (France) and published in two ESO meetings (O'ctober 1991 and April 1992).

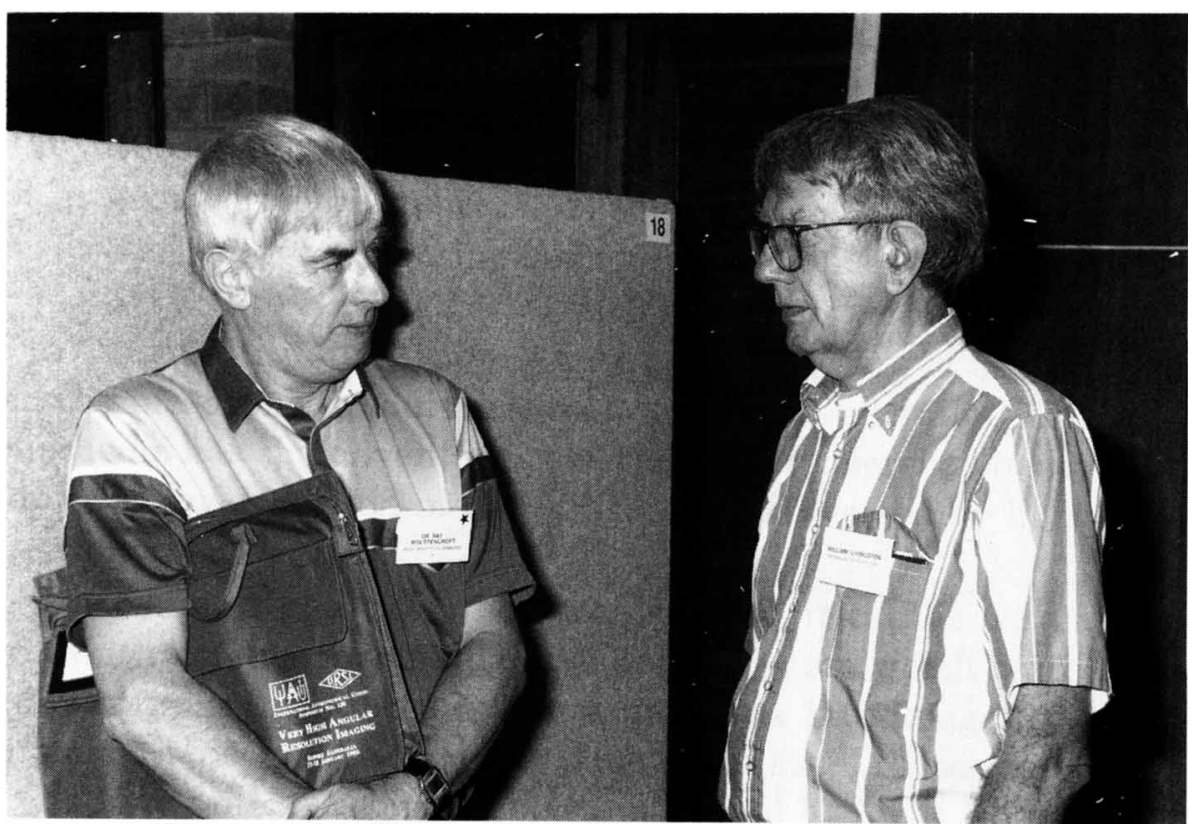

